\title{
Caracterização Sociodemográfica e Clínica de Pessoas com Estomia em Teresina
}

\section{Sociodemographic and Clinic Characterization of People with Ostomy in Teresina}

\author{
Caracterización Sociodemográfica y Clínica de Personas con Ostomía en Teresina
}

\author{
Sara Machado Mirandaํ, Maria Helena Barros Araújo Luz², Helena Megumi Sonobe, \\ Elaine Maria Leite Rangel Andrade ${ }^{4}$, Elaine Cristina de Carvalho Moura ${ }^{5}$
}

\begin{abstract}
RESUMO
O estudo teve por objetivo caracterizar o perfil sociodemográfico e clínico de pessoas cadastradas no Programa de Estomizados de Teresina (PI). Pesquisa exploratório-descritiva, transversal, com abordagem quantitativa, cujos dados foram coletados no período de junho a julho de 2013, sendo utilizado o programa Statistical Package for the Social Sciences (SPSS), versão 19.0, para análise estatística. A pesquisa foi aprovada pelo Comitê de Ética e Pesquisa (CEP) da Universidade Federal do Piauí (UFPI), sob CAAE nº 10487813.3.0000.5214. A amostra foi constituída por 107 estomizados, que atenderam aos critérios de inclusão. Houve predomínio de estomizados do sexo masculino (55,1\%); média de idade de 59,2 anos; casados (48,6\%); com filhos (86,9\%); católicos (81,3\%); renda familiar entre 1 e 3 salários mínimos (60,7\%); ensino fundamental incompleto (32,7\%); aposentados (57,0\%). As neoplasias constituíram a principal causa da estomia (71,0\%), seguidas de doenças inflamatórias intestinais (20,6\%); a maioria era colostomizada (74,8\%), permanente (48,6\%), há menos de um ano (39,3\%); apresentava efluente de consistência pastosa (74,8\%); utilizava bolsa coletora de peça única (94,4\%), sendo 100\% drenável, fornecida pelo Sistema Único de Saúde (SUS) (73,8\%). Dentre as 41 (38,3\%) complicações apresentadas pelos participantes, 30 (73,1\%) referiam-se à pele periestoma caracterizado, 29, ao eritema (96,7\%), e 11 (26,82 \%), ao estoma, sendo verificados prolapso, 10 (90,0\%), e sangramento, 1 (3,3\%). Os resultados obtidos mostraram aproximações e semelhanças em comparação com as literaturas nacional e internacional. Conclui-se que o conhecimento do perfil dessa clientela possibilitará a adequação do planejamento da assistência especializada e da provisão de equipamentos coletores e adjuvantes no contexto do Estado do Piauí.
\end{abstract}

DESCRITORES: Estomaterapia. Estomia. Enfermagem. Perfil de saúde. Neoplasias.

\begin{abstract}
The study aimed to characterize the sociodemographic and clinical profile of people registered in the ostomy program of Teresina (PI). Exploratory-descriptive, cross-sectional survey with a quantitative approach, whose data were collected between June and July 2013, by using the Statistical Package for the Social Sciences (SPSS) 19.0 software for statistical analysis. The study was approved by the Research Ethics Committee (CEP) of the Federal University of Piauí (UFPI) under CAAE n. 10487813.3.0000.5214. The sample consisted of 107 ostomy who met the inclusion criteria, through interviews using an instrument adapted from a previously developed. The results showed that the majority of ostomy (55.1\%) are male; mean age 59.2 years; married (48.6\%); with children (86.9\%); Catholic (81.3\%); family income
\end{abstract}

\footnotetext{
${ }^{1}$ Enfermeira. Mestre do Programa de Pós-Graduação em Enfermagem da Universidade Federal do Piauí (UFPI) - Teresina (PI), Brasil. Endereço para correspondência: Avenida Senador José Cândido Ferraz, 1770, Condomínio Pallazzo Maggiore 1603 - Jóquei Club - CEP: 64049-250- Teresina (PI), Brasil - E-mail: sarammiranda2@gmail.com

${ }^{2}$ Doutora em Enfermagem pela Escola de Enfermagem Anna Nery da Universidade Federal do Rio de Janeiro (UFRJ) - Rio de Janeiro (RJ), Brasil. Docente do Programa de Pós-Graduação em Enfermagem da UFPI - Teresina (PI), Brasil. Pós-graduada em Estomaterapia pela Universidade de São Paulo (USP) - São Paulo (SP), Brasil.

${ }^{3}$ Doutora em Enfermagem pela Escola de Enfermagem de Ribeirão Preto da Universidade de São Paulo (EERP-USP). Docente do Programa de Pós-Graduação Enfermagem Fundamental da EERP-USP - Ribeirão Preto (SP), Brasil. Pós-graduada em Estomaterapia pela USP - São Paulo (SP), Brasil. ${ }^{4}$ Doutora em Enfermagem pela EERP-USP - São Paulo (SP), Brasil. Docente do Programa de Pós-Graduação em Enfermagem da UFPI - Teresina (PI), Brasil. ${ }^{5}$ Doutora em Enfermagem Fundamental pela EERP-USP - Ribeirão Preto (SP), Brasil. Docente do Programa de Pós-Graduação em Enfermagem da UFPI - Teresina (PI), Brasil.

Artigo recebido em: 06/10/2014 - Aceito para publicação em: 02/07/2015
} 
between 1 and 3 minimum wages (60.7\%); have incomplete primary education (32.7\%) and retired (57.0\%). Neoplasms are the main cause of the stoma (71.0\%), followed by inflammatory bowel disease (20.6\%); colostomy (74.8\%), standing (48.6\%), with length of stay less than 1 year (39.3\%); present effluent pasty consistency (74.8\%); using one-piece pouch (94.4\%) draining (100\%) and supplied by SUS (73.8\%). Among the 41 (38.3\%) complications presented by the participants, $30(73.1 \%)$ referred to the peristoma skin characterized by erythema 29 (96.7\%) and 11 (26.82\%) to the stoma, prolapse and 10 (90.0\%) and bleeding 1 (3.3\%). The results show similarities and similarities in some aspects and differences in others, found in national and international literature. It is concluded that the knowledge produced reflects the state of health of the individual who undergoes surgery to make the stoma, the structure of the support services provided in the context of the State of Piauí.

DESCRIPTORS: Stomatherapy. Ostomy. Self-care. Nursing. Health profile. Neoplasms.

\section{RESUMEN}

El estudio tuvo como objetivo caracterizar el perfil sociodemográfico y clínico de las personas inscritas en el programa de ostomía de Teresina (PI). Encuesta exploratorio-descriptivo, transversal, con abordaje cuantitativo, cuyos datos fueron recogidos entre junio-julio de 2013, mediante el software Statistical Package for the Social Sciences (SPSS) 19.0 para el análisis estadístico. El estudio fue aprobado por el Comité de Ética en Investigación (CEP) de la Universidad Federal de Piauí (UFPI), bajo CAAE n 10487813.3.0000.5214. La muestra consistió de 107 ostomizados, según los criterios de inclusión. La mayoría de los participantes eran hombres (55,1\%); edad media de 59,2 años; casados (48,6\%); con los hijos (86,9\%); católica (81,3\%); renta familiar 1 hasta 3 sueldos mínimos (60,7\%); educación básica incompleta (32,7\%) y jubilados (57,0\%). Neoplasias son la causa principal del estoma (71,0\%), seguida de la enfermedad inflamatoria intestinal (20,6\%); colostomía (74,8\%), definitivo (48,6\%), convivio con estoma de menos de 1 año (39,3\%); con efluente pastoso (74,8\%); uso de equipo de una pieza (94,4\%), drenable (100\%) y suministrado por el Sistema Único de Salud (SUS) (73,8\%). Entre los 41 $(38,3 \%)$ las complicaciones presentadas por los participantes, 30 (73,1\%) se refirió a la piel peristoma caracterizado por eritema 29 (96,7\%) y 11 (26,82\%) al estoma, prolapso y 10 (90,0\%) y sangrado 1 (3,3\%). Los resultados son semejantes y diferentes comparados con literatura nacional y internacional. Se concluye que el conocimiento del perfil de esta clientela posibilita adecuar el planeamiento de la asistencia especializada y la provisión de equipos de ostomía y adyuvantes en el contexto del Estado de Piauí.

DESCRIPTORES: Estomaterapia. Ostomía. Autocuidado. Enfermería. Perfil de salud. Neoplasias.

\section{INTRODUÇÃO}

Nos últimos anos, as mudanças sociodemográficas, econômicas e epidemiológicas ocasionadas por urbanização acelerada, novos padrões de consumo, globalização do conhecimento, avanços científicos e tecnológicos promoveram grande impacto em âmbito nacional, com declínio da taxa de natalidade, aumento da expectativa de vida populacional e, consequentemente, da longevidade, paralelamente associado ao aumento de doenças crônicas não transmissíveis, elevado número de acidentes de trânsito e violência urbana, resultando no crescente aumento do número pessoas com estomias no Brasil, sendo que a cada ano são realizados, aproximadamente, 1 milhão e 400 mil procedimentos cirúrgicos com confecção de estomias ${ }^{1}$.

Na maioria das vezes, as estomias estão vinculadas às doenças crônicas intestinais e urinárias, como os cânceres colorretal e de bexiga, às doenças inflamatórias, como doença de Crohn, retocolite ulcerativa e diverticulite, e ao tratamento de enfermidades congênitas e trauma abdominal ${ }^{2}$. No Brasil, dentre as principais causas relacionadas à necessidade de estomia na população adulta e idosa, encontram-se as neoplasias, principalmente o câncer colorretal, cuja estimativa mais recente pelo Instituto Nacional de Câncer José Alencar Gomes da Silva (INCA) foi para o ano de 2014, com 32.600 casos, sendo 15.070 homens e 17.530 mulheres ${ }^{3}$.

Considerando o aumento da população estomizada, ressaltamos conquistas importantes em relação à assistência especializada e à implementação de políticas públicas para assegurar a acessibilidade para essa clientela, como o movimento, na década de 1970, marcado pela forte presença dos estomizados, com o apoio dos profissionais de saúde, especialmente médicos coloproctologistas e enfermeiros, a criação da primeira associação de estomizados, em 1975, em Fortaleza (CE), e, em 1990, a implementação do primeiro curso de especialização em estomaterapia no Brasil, visando formar enfermeiros para prestar assistência avançada em resposta às reivindicações da clientela. Posteriormente, em 1992, foi criada a Associação Brasileira de Estomaterapia: estomias, feridas e incontinências (SOBEST), que fortaleceu ainda mais o movimento ${ }^{4}$. 
Houve a criação e a implementação de políticas públicas governamentais específicas para as pessoas com estomia, como a Portaria SAS/MS no 400, de 16 de novembro de 2009, que estabeleceu diretrizes nacionais para a Atenção à Saúde de pessoas estomizadas no âmbito do Sistema Único de Saúde (SUS) e definiu os três níveis de atenção prestados às pessoas com estoma, além das responsabilidades com a promoção da saúde, a assistência, a reabilitação e as respectivas atribuições. Além disso, propõe modelos de estruturas físicas adequadas para realizar o atendimento e a descrição dos materiais e equipamentos coletores necessários ao cuidado integral às pessoas com os estomas pelo SUS, por uma equipe interdisciplinar, e os fluxos para os serviços de referência e contrarreferência ${ }^{5,6}$.

A assistência de enfermagem às pessoas com estomias perpassa pela necessidade de integração dos diferentes níveis de atendimento à saúde, como as instituições hospitalares $\mathrm{e}$ os ambulatórios de especialidades, pelas repercussões físicas e psicossociais do processo de estomização e pela necessidade de aprendizagem do autocuidado com a estomia, equipamentos coletores e adjuvantes, que são aspectos cruciais durante o seguimento no Programa de Ostomizados para a reabilitação dessas pessoas. Com a aprendizagem do autocuidado, a pessoa com estomia adquire maior autonomia, maior capacidade de desempenhar atividades necessárias à saúde, além de enfrentar e conviver com as repercussões e limitações resultantes das alterações de saúde ${ }^{1}$.

No Programa de Ostomizados, o enfermeiro realiza avaliação clínica da estomia e da região periestoma para realizar a indicação do equipamento e adjuvante adequados para cada pessoa, considerando aspectos físicos, atividades diárias desenvolvidas por estas e capacidade para o autocuidado, o que previne desenvolvimento de lesões periestomas, proporciona maior segurança e favorece a adaptação à nova condição, gerando, dessa forma, melhor custo-benefício ao sistema público de saúde local ${ }^{1,6}$.

Apesar de muitos avanços em relação à implementação de políticas públicas específicas para essa clientela, o desafio nesse programa de saúde é atender à demanda de necessidades de cuidados especializados, assim como a previsão e a provisão de equipamentos coletores e adjuvantes, para potencializar o alcance de melhores resultados ${ }^{1,6}$

Assim, acreditamos que para a operacionalização dessas políticas é necessário conhecer as características sociodemográficas e clínicas dessa clientela, para otimizar os recursos humanos e materiais.

\section{OBJETIVO}

Caracterizar o perfil sociodemográfico e clínico de pessoas cadastradas no Programa de Atenção às Pessoas com Estomia de Teresina (PI).

\section{MÉTODOS}

Trata-se de um estudo descritivo com abordagem quantitativa dos dados acerca da caracterização do perfil sociodemográfico e clínico de pessoas com estomias cadastradas no Programa de Atenção às Pessoas com Estomia de Teresina (PI).

A amostra foi não probabilística, constituída por 107 pessoas cadastradas no programa até junho de 2013, sendo que 97 apresentavam estomia intestinal, e 10, estomia urinária. A coleta de dados ocorreu no período de junho a julho de 2013 por meio de entrevistas previamente agendadas, em que se utilizou formulário semiestruturado, com perguntas abertas e fechadas, preenchidas pelo entrevistador com os estomizados que atenderam aos critérios de inclusão: estar cadastrado no Centro Integrado de Saúde Lineu Araújo (CISLA), ter idade acima de 18 anos, ambos os sexos, residir no município de Teresina e aceitara participar da pesquisa assinando o Termo de Consentimento Livre e Esclarecido. As entrevistas foram digitadas no Microsoft ${ }^{\circledR}$ Excel e transportadas para o programa Statistical Package for the Social Sciences (SPSS), e, em seguida, foi realizada a análise descritiva dos dados. A pesquisa foi aprovada pelo Comitê de Ética e Pesquisa (CEP) da Universidade Federal do Piauí (UFPI), sob CAAE no 10487813.3.0000.5214, de acordo com a Resolução 466/12 do Conselho Nacional de Saúde.

\section{RESULTADOS}

A caracterização sociodemográfica dos 107 estomizados participantes do estudo mostra que a maioria, $59(55,1 \%)$, é do sexo masculino, cuja faixa etária predominante foi de 60 a 80 anos, com 47 (43,9\%), apresentando média de 59,3 anos de idade, casada/em união estável, 52 (48,6\%), e possuía um ou mais filhos, 93 (86,9\%). 
Predominaram os que possuíam ensino fundamental incompleto, 35 (32,7\%), renda familiar de 1 a 3 salários mínimos, 65 (60,7\%), condição previdenciária como aposentados, 61 (57,0\%), moravam em casa própria, 88 (82,2\%), e se declararam católicos, 87 (81,3\%).

Quanto à causa básica que resultou na construção da estomia, verificou-se que a maioria, $76(71,0 \%)$, foi por neoplasias, sendo 80 colostomias (74,8\%), 15 (14\%) ileostomias e $10(9,3 \%)$ urostomias, e $2(1,9 \%)$ possuíam dois tipos de estoma (colostomia e urostomia).

Em relação à coloração do estoma, a maioria, 104 (97,2\%), apresentou cor róseo-avermelhada, com forma redonda, $63(58,9 \%)$, protrusa, 75 (70,1\%), plana, 23 (21,5\%), e retraída, $9(8,4 \%)$. Quanto ao tempo de permanência, a maioria, $52(48,6 \%)$, apresentou estomia permanente, com localização da estomia no quadrante inferior esquerdo, $56(52,3 \%)$, $40(37,4 \%)$ no quadrante inferior direito, $3(2,8 \%)$ no quadrante superior esquerdo e $8(7,5 \%)$ no quadrante superior direito, sendo observado que a maioria, $80(74,8 \%)$, apresentou efluente pastoso, seguido por $24(22,4 \%)$ com forma líquida e 3 (2,8\%) com consistência sólida.

Por outro lado, o tipo de equipamento coletor utilizado pela maioria dos estomizados, 101 (94,4\%), era bolsa com peça única e apenas 6 (5,6\%) utilizavam dispositivo de duas peças; quanto à abertura, $100 \%$ das bolsas eram drenáveis ou abertas.

Verificou-se que dos 107 participantes, 41 (38,31\%) apresentaram complicações no estoma ou na pele periestoma, sendo $11(26,82 \%)$ complicações de estoma, 10 (90,0\%) apresentaram prolapso e $1(9,0 \%)$ com sangramento. Dos $30(73,1 \%)$ estomizados que apresentaram complicações na pele periestoma, 29 (96,7\%) tiveram eritema, e 1 (3,3\%), outra complicação.

\section{DISCUSSÃO}

Em relação à análise das variáveis sociodemográficas e econômicas dos estomizados participantes da pesquisa, pode-se verificar o predomínio de pacientes do sexo masculino, idosos, casados, com filhos, aposentados, católicos, cuja formação educacional foi o ensino fundamental incompleto, com renda de até 3 salários mínimos e que residiam em casa própria, o que corrobora resultados de outros estudos que indicam constituição familiar nuclear e condição socioeducacional e econômica predominante na população brasileira, com a maioria composta por estomizados do sexo masculino $(52,6 \%)$ e média de idade de 61,6 anos $^{7,8}$. Isso pode ser decorrente do aumento da violência urbana e dos acidentes de trânsito, associados ao uso de drogas lícitas e ilícitas, tornando o sexo masculino mais exposto a riscos de trauma abdominal, outras doenças e agravos. Ainda, a população masculina utiliza com menor frequência os serviços de saúde e as medidas preventivas, o que leva tal população a procurar o atendimento médico tardiamente, quando apresenta manifestações do agravamento das doenças. Além disso, o sexo masculino possui certa invisibilidade nos serviços de atenção primária, fator que faz com que os homens se privem de medidas de promoção da saúde e prevenção de doenças, o que pode torná-los mais vulneráveis a agravos e complicações, por isso o diagnóstico em estágio avançado ${ }^{9,10}$.

Apesar de a maioria ser casada, a confecção da estomia e a convivência com o equipamento coletor geram sentimentos conflituosos, bem como alterações sexuais, que podem desgastar os relacionamentos afetivos, em decorrência de negação, diminuição da autoestima, sensação de mutilação e rejeição própria e dos familiares ${ }^{11}$.

No que diz respeito ao nível de escolaridade, assim como em outros estudos com a mesma clientela, predominou ensino fundamental incompleto ${ }^{7,12}$. Tal resultado revela um perfil preocupante em relação à cidadania e aos direitos, pois quanto mais baixa a escolaridade, maior a dificuldade para questionar os profissionais a respeito do problema de saúde e realizar os cuidados necessários, além de comprometer a implementação de ações educativas de autocuidado ${ }^{1}$.

O baixo nível de escolaridade pode ser um fator preponderante para a não prevenção do câncer colorretal, uma das principais causas básicas para a confecção das estomias, devido à ausência de conhecimento sobre a necessidade de realizar exames de rotina para a detecção precoce do câncer, e manutenção de hábitos prejudiciais, como etilismo, tabagismo, sedentarismo, maus hábitos alimentares, fatores intimamente ligados ao surgimento de neoplasias malignas ${ }^{13}$.

Em relação à renda familiar, este estudo coincide com o percentual encontrado em outras regiões, nas quais a média salarial é compreendida entre 1 e 2 salários mínimos $(66,51 \%)^{12}$.

No tocante à religião, o predomínio de católicos foi semelhante aos resultados de outros estudos ${ }^{7,14}$. É sugestivo que os estomizados busquem nas atividades espirituais subsídios para o enfrentamento dos seus problemas físicos e psicológicos, vinculados ao estoma.

Em relação à condição previdenciária, a maioria encontra-se inativa, fato que pode ser observado não somente pelo 
uso da estomia, mas também pela faixa etária dos participantes ( $>60$ anos), podendo estes pacientes ser aposentados pela idade, e não pela presença da estomia.

Verifica-se que é comum, depois da cirurgia, o paciente aposentar-se, deixando de ser provedor da família e tornando-se dependente em relação ao seu cuidado. Os limites ou a perda da capacidade para o trabalho são definidos, na maioria das vezes, pelo próprio estomizado, que se classifica como alguém que está impossibilitado de ser útil à sociedade ${ }^{14}$.

No que diz respeito ao tipo de moradia, a grande maioria afirmou residir em casa própria. Pode-se observar que, mesmo com a maior parte dos entrevistados apresentando baixo poder aquisitivo, entre 1 e 3 salários mínimos, os pacientes possuem casa própria, fato que amplia a visibilidade da política nacional de habitação, que beneficia as pessoas de baixa renda.

Verificou-se que as neoplasias são a maior causa de estomia, seguidas das doenças inflamatórias. Um estudo realizado recentemente em Minas Gerais corrobora os resultados desta pesquisa, em que as neoplasias malignas representaram $(75 \%)$ das causas de estomia ${ }^{8}$. No que diz respeito às doenças inflamatórias, uma pesquisa feita também em Teresina, no ano de 2009, apontava que $10,5 \%$ das estomias eram por essa causa ${ }^{7}$.

Pode-se observar que as causas das estomias condizem com a diversidade de idades dos estomizados do estudo, que engloba jovens, adultos e idosos. Entre os jovens e adultos, evidencia-se maior frequência de doenças inflamatórias intestinais, tais como a doença de Crohn e a retocolite ulcerativa, além de traumas decorrentes da violência urbana. Entre os idosos, verifica-se a predominância de quadros clínicos obstrutivos relacionados ao câncer colorretal e a doenças inflamatórias intestinais, como diverticulite e volvo.

No tocante ao tipo de estomia realizada, houve predominância de colostomia, seguida de ileostomia. Em um estudo realizado também em Teresina foram obtidos resultados semelhantes, em que a colostomia foi de $84,1 \%$ e ileostomia foi de $15,7 \%{ }^{7}$. A colostomia é a intervenção cirúrgica geralmente realizada nos casos em que o paciente tem neoplasia intestinal ${ }^{10}$.

Em relação à coloração da estomia, a maioria foi rósea, com formato de estoma redondo, seguido pelo formato irregular. O formato redondo diminui as complicações, principalmente por favorecer a adaptação do equipamento coletor e evitar extravasamento de efluentes.
Os estomas com aparência normal devem apresentar formato regular, coloração róseo-avermelhada, brilhante, úmido, com pele periestoma íntegra. A umidade e a coloração indicam fluxo sanguíneo adequado para a área ${ }^{15}$.

Em relação ao tipo de implantação na pele, a maior parte foi composta pelo modelo protruso, seguido pelo plano. A protrusão recomendada de um estoma é de $3 \mathrm{a} 5 \mathrm{~cm}$, para evitar lesões de pele por vazamento de efluente. No Reino Unido, em 2005, foi realizada uma auditoria em estomaterapia que estabeleceu que a altura mínima para a confecção de estomias era de $5 \mathrm{~mm}$ para colostomia e de $20 \mathrm{~mm}$ para ileostomia, para evitar complicações ${ }^{15,16}$.

Quanto à temporalidade da estomia, a maioria foi permanente. A permanência ou não das estomias está diretamente relacionada com o diagnóstico de indicação de sua confecção. A definitiva está associada frequentemente aos cânceres colorretal e urogenital, e a temporária, aos traumas. Em outros estudos, foram observados resultados semelhantes, pois $51,24 \%$ apresentaram estomia permanente e $48,76 \%$ possuíam estomia temporária ${ }^{12}$.

No tocante à localização, a maioria dos estomas localizava-se no quadrante inferior esquerdo, seguida por quadrante inferior direito. Somando as estomias localizadas nos quadrantes do lado esquerdo obtém-se a maioria, fato que explica a predominância do diagnóstico oncológico e do tipo de efluente pastoso.

A consistência do efluente varia conforme a localização de confecção do estoma; assim, colostomias sigmoide e descendente apresentam efluentes sólidos não corrosivos; colostomia transversa tem efluente de consistência semissólida não corrosiva; colostomia ascendente tende a apresentar efluentes semilíquidos e corrosivos. Vale ressaltar que a emissão de gases e odores tende a aumentar em colostomias transversas e ascendentes ${ }^{8}$.

Quanto à abertura da bolsa coletora, predominaram o tipo drenável e o equipamento de uma peça, em decorrência da padronização de equipamentos realizada nesse do serviço, limitando o processo de licitação da compra e o fornecimento de outros modelos de equipamentos coletores, sendo mais recomendado o modelo de duas peças, com protetor de pele, para prevenção de complicações.

Outros estudos que abordaram o uso de equipamentos coletores corroboram esta pesquisa, em que $66,6 \%$ dos participantes usavam bolsa coletora drenável, e 91,6\%, sistema de uma peça ${ }^{15}$. A bolsa deve ser esvaziada quando atingir um terço do conteúdo ou, no máximo, metade de 
sua capacidade, pois a durabilidade e a adesividade da base da bolsa coletora à pele serão maiores ${ }^{17,18}$.

A indicação de equipamentos e adjuvantes aos pacientes depende do tipo de estoma e da consistência do efluente, assim como da confecção cirúrgica, além da presença de complicações, nível de protrusão, capacidade para o autocuidado, tipo de atividade do paciente, barreira de proteção ou protetores cutâneos disponíveis ${ }^{19}$.

Das complicações apresentadas no estoma e na pele periestoma, a maioria foi composta por prolapso e eritema. Em estudos semelhantes, 50,6\% dos pacientes apresentaram complicações, predominando as dermatites, com $28,7 \%$, fato que diverge das características deste estudo ${ }^{10}$.

O prolapso é uma complicação nos estomas terminais, com maior frequência nos estomas em alça, em decorrência de exteriorização do segmento intestinal móvel, distante dos pontos de fixação anatômicos (ângulo hepático, ângulo esplênico, ângulo descendente-sigmoide $)^{17}$. Tal situação decorre de dificuldades para a confecção da estomia e anomalia da estrutura abdominal, gerando grande desconforto abdominal e dificuldade no manejo da estomi ${ }^{20}$.Já as lesões de pele periestoma, como as dermatites, são ocasionadas por uso ou indicação inadequada dos equipamentos, corte excessivo do orifício da barreira protetora, deixando a pele exposta à ação do efluente ${ }^{21}$.

Destacamos a importância do uso do cinto para a prevenção do prolapso em pacientes com abdômen globoso ou que realizam atividade física e no tratamento daqueles que apresentam prolapso, para evitar o agravamento. Em relação ao eritema, deve-se enfatizar a proteção da pele, o ensino do autocuidado e a avaliação periódica. Essas adequações de indicações de equipamentos somente é possível com a caracterização do perfil de saúde dessa clientela, para otimizar a gestão especializada dos recursos materiais e humanos.

O aparecimento das complicações é consequência de consultas pré-operatórias precárias, em especial da falta de demarcação de estoma no pré-operatório, pois a má localização dificulta o autocuidado, a visualização da pele e a troca de equipamentos. A má adaptação da base do equipamento à pele deve-se principalmente à escolha inadequada do local de confecção da estomia na parede abdominal, ou seja, proximidade às depressões, pregas cutâneas, proeminências ósseas, entre outros acidentes anatômicos que poderão resultar em vazamento do conteúdo drenado pela estomia ou impossibilidade de adesividade da base do equipamento na pele ${ }^{22}$.

As limitações deste estudo estão relacionadas com a deficiência nos registros de informações, a falta de organização e a atualização do banco de dados no programa de saúde, além da falta de equipamento coletor para distribuição e dificuldade de deslocamento das pessoas com estoma intestinal, que, para terem acesso aos equipamentos, enviavam terceiros para recebê-los e cuja ausência nas reuniões aumenta a prevenção de complicações como eritema e prolapso, identificadas neste estudo.

\section{CONCLUSÃO}

Com o perfil sociodemográfico e clínico das pessoas estomizadas residentes em Teresina (PI), identificamos que a maioria era composta por pacientes do sexo masculino, com idade ente 60 e 80 anos, casados ou mantinham união estável, possuíam ensino fundamental incompleto ou eram analfabetos, renda familiar de até 3 salários mínimos, praticantes do catolicismo, aposentados e residiam em casa própria.

No que diz respeito à causa básica que deu origem às estomias, verificou-se que a maioria das cirurgias estomas foi ocasionada pela neoplasia.

A maioria possuía colostomia, sendo permanente localizada no quadrante inferior esquerdo, com efluente pastoso, de formato redondo, coloração róseo-avermelhada e implantação protrusa na pele, fazia uso de equipamento coletor drenável e de peça única.

Acredita-se que este estudo trouxe maior visibilidade sobre a clientela estomizada atendida para o poder público e para os profissionais de saúde, o que possibilitará traçar estratégias mais eficientes para prevenção, tratamento e reabilitação, com melhoria da qualidade de vida desses pacientes, além da contribuição para o dimensionamento de equipamentos e adjuvantes nesse programa. 


\section{REFERÊNCIAS}

1. Lenza NFB, Sonobe HM, Buetto LS, Santos MG, Lima MS. O ensino do autocuidado aos pacientes estomizados e seus familiares: uma revisão integrativa. Rev Bras Promoção a Saúde. 2013;26(1):139-45.

2. Silva AC, Silva GNS, Cunha RR. Caracterização de pessoas estomizadas atendidas em consulta de enfermagem do serviço de estomoterapia do município de Belém-PA. Rev. ESTIMA. 2012;10(1):20-7.

3. Instituto Nacional de Câncer (INCA) [Internet]. [cited 2014 Apr 10]. Available from: http://www2.inca.gov.br/wps/wcm/ connect/tiposdecancer/site/home/colorretal/definicao+

4. Geraldi I. Associação Brasileira de Estomaterapia - SOBEST. Campinas: Editora Pinti; 2012.

5. Brasil. Ministério da Saúde. Secretaria de Atenção à Saúde. Política Nacional de Saúde da Pessoa com Deficiência. 1 a ed. Brasília: Ministério da Saúde; 2010.

6. Brasil. Ministério da Saúde. Secretaria de Atenção à Saúde. Portaria n 400, de 16 de novembro de 2009 [Internet]. Brasília: Ministério da Saúde; 2009. [cited 2013 May 23]. Available from: http://www.mprs.mp.br/areas/dirhum/ arquivos/p_sas_400_2009_ostomizados.pdf

7. Luz MHBA, Andrade DS, Amaral HO, Bezerra SMG, Benício CDAV, Leal ACA. Caracterização dos pacientes submetidos a estomas intestinais em um hospital público de Teresina-PI. Texto Contexto - Enferm. 2009;18(1):140-6.

8. Fernandes RM, Miguir ELB, Donoso TV. Perfil da clientela estomizada residente no município de Ponte Nova, Minas Gerais. Rev Bras Colo-proctol. 2010;30(4):385-92.

9. Figueiredo W. Assistência à saúde dos homens: um desafio para os serviços de atenção primária [Internet]. Ciênc Saúde Coletiva. 2005;10(1):104-9. [cited 2014 Feb 25]. Available from: http://www.scielo.br/scileo.php?script=sci_ arttext\&pid=\$1413-81232005000100017\&nrm=iso

10. Santos CHM, Bezerra MM, Bezerra FMM, Paraguassu BR. Perfil do paciente ostomizado e complicações relacionadas ao estoma. Rev Bras Colo-proctol. 2007;27(1):16-9.

11. Batista MRFF, Rocha FCV, Silva DMG, Silva FJG Jr. Autoimagem de clientes com ostomia em relação à bolsa coletora [Internet]. Rev Bras Enferm. 2011;64(6):1043-7. [cited 2014 Mar 01]. Available from: http://www.scielo.br/pdf/reben/v64n6/v64n6a09.pdf
12. Silva AC, Silva GNS, Cunha RR. Caracterização de pessoas estomizadas atendidas em consulta de enfermagem do serviço de estomoterapia do município de Belém-PA. Rev. ESTIMA. 2012;10(1):20-7.

13. Guarisi M. Rastreamento, Diagnóstico e tratamento das lesões Precursoras e do câncer Invasor do Colo Uterino no município de Franco da Rocha, SP [Internet]. Rev Bras Cancerol. 2004;50(1):7-15. [cited 2014 Apr 23]. Available from: http://www.inca.gov.br/rbc/n/n_50/v01/pdf/ ARTIGO01.pdf

14. Sonobe HM, Barichello E, Zago MMF. A visão do colostomizado sobre o uso da bolsa de colostomia. Rev Bras Cancerol. 2002;48(3):341-8.

15. Vujnovich A. Pre and post-operative assessment of patient with a stoma [Internet]. Nursing Standart. 2001;22:121-9. [cited 2013 Oct 20]. Available from: http://rcnpublishing. com/doi/abs/10.7748/ns2008.01.22.19.50.c6315

16. Cottam J, Richards K. National audit of stoma complications within 3 weeks of surgery [Internet]. Gastrointestinal Nursing. 2006;4(8):34-9. [cited 2013 Oct 20]. Available from: http://www.internurse.com/cgibin/go.pl/library/article.cgi?ui d=22084; article=gn_4_8_34_39;format=pdf

17. Crema E, Silva R. Estomas: uma abordagem interdisciplinar. Uberaba: Editora Pinti; 1997.

18. Netina SMB. Prática de enfermagem. 7a ed. Rio de Janeiro: Guanabara Koogan; 2007.

19. Cesaretti, IUR. Dermatite Periostomal: da etiologia ao tratamento e assistência de enfermagem. Acta Paul Enferm. 1997;10(2):80-7.

20. Dukes S. Considerations when caring for a person with a prolapsed stoma. Br J Nurs. 2011;19(17):1-5.

21. Williams J. Evaluating skin care problems in people with stomas [Internet]. Br J Nurs. 2010;19(17):6-15. [cited 2014 Feb 10]. Available from: http://ncbi.nlm.nih.gov/ pubmed/20871505

22. Manjoubi B, Goodarki, KK, Mohammad-Sadegui H. Quality of life in stomas patients: appropriate and inappropriate stomas sites [Internet]. World J Surg. 2010;34(1):147-52. [cited 2013 Apr 22]. Available from: http://www.ncbi.nlm.nih. gov/pubmed/19936980 